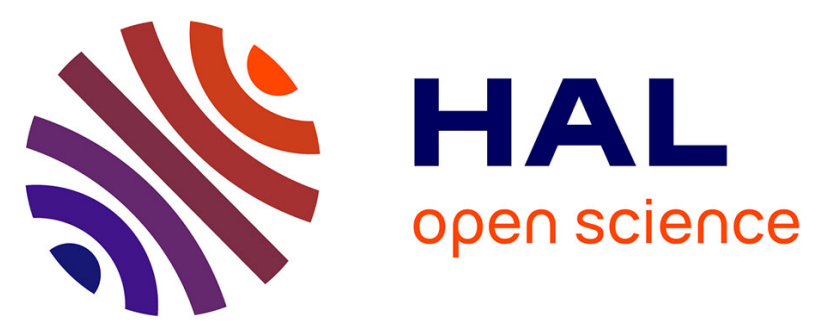

\title{
Statistical Analysis of Guided Wave Imaging Algorithms Performance Illustrated by a Simple Structural Health Monitoring Configuration
}

Andrii Kulakovskyi, Olivier Mesnil, Bastien Chapuis, Oscar D'almeida, Alain Lhemery

\section{To cite this version:}

Andrii Kulakovskyi, Olivier Mesnil, Bastien Chapuis, Oscar D'almeida, Alain Lhemery. Statistical Analysis of Guided Wave Imaging Algorithms Performance Illustrated by a Simple Structural Health Monitoring Configuration. Journal of Nondestructive Evaluation, Diagnostics and Prognostics of Engineering Systems, 2021, 4 (3), pp.031001. 10.1115/1.4049571 . hal-03170270

\author{
HAL Id: hal-03170270 \\ https://hal.science/hal-03170270
}

Submitted on 18 Mar 2021

HAL is a multi-disciplinary open access archive for the deposit and dissemination of scientific research documents, whether they are published or not. The documents may come from teaching and research institutions in France or abroad, or from public or private research centers.
L'archive ouverte pluridisciplinaire HAL, est destinée au dépôt et à la diffusion de documents scientifiques de niveau recherche, publiés ou non, émanant des établissements d'enseignement et de recherche français ou étrangers, des laboratoires publics ou privés. 


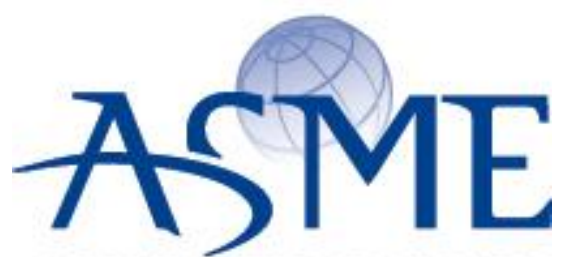

SETTING THE STANDARD

\section{American Society of Mechanical Engineers}

ASME Accepted Manuscript Repository

Institutional Repository Cover Sheet

ASME Paper Title: Statistical analysis of guided wave imaging algorithms performance illustrated by a simple

structural health monitoring configuration

Authors: $\quad$ Andrii Kulakovskyi, Olivier Mesnil, Bastien Chapuis, Oscar d'Almeida, Alain Lhémery

ASME Journal Title: Journal of Nondestructive Evaluation

Volume/Issue $4(3)$

Date of Publication (VOR* Online): February $3^{\text {rd }}, 2021$

https://asmedigitalcollection.asme.org/nondestructive/article/4/3/031001/1094104/S

ASME Digital Collection URL: Analysis-of-Guided-Wave-Imaging

DOI: $\quad$ https://doi.org/10.1115/1.4049571

*VOR (version of record) 


\title{
Statistical analysis of guided wave imaging algorithms performance illustrated by a simple SHM configuration
}

\author{
Andrii Kulakovskyi \\ Olivier Mesnil* \\ Université Paris-Saclay, CEA, List Université Paris-Saclay, CEA, List, France \\ and Safran Tech, France \\ Bastien Chapuis \\ Université Paris-Saclay, CEA, List, France Safran Tech, France \\ Oscar d'Almeida
Safran Tech, France \\ Alain Lhémery
Université Paris-Saclay, CEA, List, France
}

Guided Wave-based Structural Health Monitoring (GWsSHM) system aims at determining the integrity of a wide variety of plate-like structures such as aircraft fuselages, pipes and fuel tanks. It is often based on a sparse grid of piezoelectric transducers for exciting and sensing guided waves $(G W s)$ that under certain conditions interact with damage while propagating. In recent years, various defect imaging algorithms have been proposed for processing GWs signals, and, particularly, for computing an image representing the integrity of the studied structure. The performance of GWsSHM system highly depends on a signal processing methodology. This paper compares defect localization accuracy of the three state-of-art defect imaging algorithms (Delay-AndSum, Minimum Variance, and Excitelet) applied to an extensive simulated database of GWs propagation and GWsdefect interaction in aluminum plate under varying temperature and transducers degradation. This study is conducted in order to provide statistical inferences, essential for SHM system performance demonstration.

\section{Introduction}

One of the most important challenges for engineering structure manufacturers, end-users, and maintenance teams is to evaluate the integrity of in-service structures on a frequent or continuous time basis. Applied physical loads, thermo-mechanical aging, impacts and other external factors deteriorate their mechanical properties and might lead to defects. The aerospace industry is concerned with this problematic, as well as many other industries such as oil and gas or nuclear. They are interested in estimating and extending the remaining useful life of structures, but a high level

${ }^{*}$ Corresponding author: olivier.mesnil@cea.fr of knowledge is necessary to guarantee both their availability and reliability. Although various Non-Destructive Techniques have been developed to evaluate the integrity of structures, they are usually expensive, time-consuming and often divert the structure from normal operation.

In light of this, Structural Health Monitoring (SHM) can contribute significantly towards enhancing the reliability and profitability of engineering structures $[1,2]$. SHM relies on permanently embedded transducers and knowledgebased methods, i.e., signal processing algorithms [3,4]. Embedded transducers are typically used for measuring a structural dynamic response either in a passive or active way. A knowledge-based system then processes the collected signals in order to evaluate the current health of the structure [5]. As the SHM systems are integrated ones, the inspection can be conducted anytime. They enable continuous monitoring, but, at the same time, impose additional constraints such as optimal integration and robustness against sensors aging.

Guided elastic Waves (GWs) are widely used in SHM systems for damage detection, localization, and sizing [6-8]. They can propagate over significant distances while being sensitive to both surface and subsurface defects. Therefore, they require a limited number of sensors to inspect large areas. GWs can be easily actuated and sensed by a set of piezoelectric transducers (PZTs) permanently attached to the surface or embedded within the structure. It is worth noting that GWs are multi-modal and dispersive, thus they require advanced post-processing techniques to extract defect signatures from the collected data.

One of the conventional approaches used in SHM consists in comparison of the defect-free state of the structure with the damaged one. The transducers act either as a emitters or receivers of GWs. The baseline signals are recorded from the pristine structure and subtracted from signals mea- 
sured in an unknown state [9]. If a damage occurs, the GWs propagation is disturbed by a defect in the vicinity of the GW path. The difference signals are often called residual signals and contain wavepackets scattered by the defect. Recently, several GWs Imaging (GWI) algorithms have been proposed to process residual signals in order to compute single or multi-spectral images. Compared to raw signals, such images are easy-to-analyze and provide direct information on both structural damage presence and location.

Among the wide variety of GWI techniques, [10-13] three of them are studied and compared in this work: DelayAnd-Sum (DAS) [14], Minimum Variance (MV) [15], and Excitelet (EXC) [16]. Each method processes the collected signals in order to compute an image representing the integrity of the inspected structure. The first two algorithms, DAS and MV, require the knowledge of the group velocity of the inspecting guided mode in each direction of propagation. The group velocity is computed for the central excitation frequency, so these algorithms neglect the GWs dispersion. On the other hand, Excitelet takes into account the GWs dispersion, but it requires a comprehensive understanding of the GWs propagation in the structure as well as the transducers dynamics.

The reliability of an SHM system has to be estimated before the deployment. It involves the evaluation of several aspects such as robustness to environmental effects, the Probability of Detection (PoD) and the Probability of False Alarm (PoFA) of the system $[8,17]$. In GW-SHM, different PoD and PoFA methods [18-22] have been proposed in the literature to evaluate damage detection performance. The localization performance of defect imaging algorithm constitutes an essential part of the SHM process. To the knowledge of authors, GWI results have been reported either experimentally or numerically only for a limited number of specimens. Consequently, limitations and performance of GWI algorithms either in ideal or in changing environmental conditions, have not been thoroughly studied yet.

Such limitation must be evaluated by means of statistical analysis, which requires an extensive database of GWI specimens. However, the cost of generating an extensive experimental database is prohibitive due to the large number of specimens required. Hence, numerical tools can be considered as a proper solution for the generation of large dataset, but until recently, the computation cost was too high. Newly developed finite elements tool for GWs simulation enables the creation of massive databases at a reasonable computation cost. Note that the GWI configuration studied here is fairly simple, and has already been presented extensively in the literature. The objective of this work is to present a methodology for statistical assessment of GWI algorithms so that corresponding inferences on damage localisation performance, computational complexity and imaging quality can be drawn and eventually translated to the real-world applications. The authors acknowledge the simplicity of the configuration under study, as it has been selected for the purpose of comparing imaging algorithms. Furthermore, the experimental validity of such algorithms has been demonstrated in the past, including in honeycomb composite structures [23].

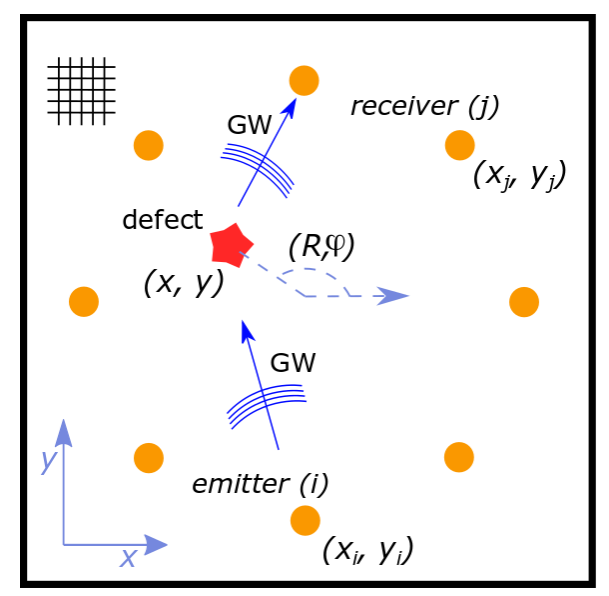

Fig. 1: Schematic representation of the Guided waves imaging methodology. Orange circles and red star define piezoelectric transducers and damage locations respectively. Blue arrows represent GW propagation in the structure.

In this paper, a statistical study on the defect localization performance under varying temperature is presented for the three GWI algorithms at the three central interrogation frequencies. The paper is organized as follows. Firstly, a brief description of the defect imaging algorithms is presented. Secondly, the simulation method for GWs propagation is presented. It is followed by the generation and description of the database of GWI results for different defect sizes and locations. Finally, the statistical analysis of the defect localization accuracy is presented. This analysis is based on the comparison of exact and estimated defect position and various statistical tools and metrics are applied to infer on the defect localization performance.

\section{Guided Waves Imaging}

Guided Wave Imaging (GWI) is a fast, reliable, and costefficient method for the damage detection, localization, and characterization in plate-like structures. It can be implemented using a sparse embedded array of inexpensive and low energy consumption piezoelectric transducers. As such, this method is a natural choice for SHM system, but the knowledge of the undamaged state of the structure, the socalled baseline, is often required.

Baseline approaches are based on the comparison between the reference signals and signals at the current time, in which the state of the structure is unknown. In light of this, various imaging algorithms have been developed to process residual signals, which are defined as the difference between the current and the baseline signals, and to compute an image representing the integrity of the structure. The damage presence and location can be directly deduced from the spatial intensity distribution of the image.

\subsection{Imaging methodology}

A schematic of the GWI process is shown in Figure 1. A grid of pixels discretizes the region of interest of the stud- 
ied specimen, where each pixel of the image is mapped to the corresponding elementary portion of the structure. In the current study, we use three algorithms, namely DAS, MV and Excitelet, which compute a Damage Index (DI) value at each pixel of the image. For a single transducer pair, each imaging algorithm maps an echo arising from the defect to an ellipse with foci at the transducers locations. Following a round-robin process, all signals are processed, and the defect location is defined at the intersection of ellipses [9].

\subsection{Defect imaging algorithms}

In general, permanently attached transducers are excited with a Hanning modulated tone burst in order to generate GWs in the structure. The central excitation frequency is usually chosen below the first cutoff frequency to reduce the complexity of GWs signals. Received signals typically contain multiple propagating modes, at least the $A_{0}$ and $S_{0}$ modes, and are additionally complicated by the GWs reflections from the edges and other structural features like flaws, rivet etc. Collected signals of the current state are subtracted from a baseline, when the structure is defect free, in order to obtain the residual signals.

\subsubsection{Delay-And-Sum algorithm}

Michaels et al. [14] proposed the DAS imaging algorithm to process residual signals using a single guided mode. Since GWs are dispersive, this algorithm is rather convenient for imaging defects that are not remote from the transducers network as a temporal resolution of the GWs degrades with the propagating distance. DAS neglects the GWs dispersion, and is mostly relevant for narrow band excitations.

The image is computed as follows. First, the envelopes of the analytical residual signals are calculated using the Hilbert transform:

$$
r_{i, j}(t)=\sqrt{u_{i, j}(t)^{2}+H\left(u_{i, j}(t)\right)^{2}}
$$

where $r_{i, j}(t)$ is an envelope of the residual signal, $u_{i, j}(t)$ is a residual signal measured by the pair of piezoelectric transducers $i, j$ and the operator $H$ denotes the Hilbert transform. Then, the time of flight of the GWs is computed as follows:

$$
\begin{aligned}
t_{i, j}(x, y)=\frac{\sqrt{\left(x-x_{i}\right)^{2}+\left(y-y_{i}\right)^{2}}}{C_{g}}+ \\
\frac{\sqrt{\left(x-x_{j}\right)^{2}+\left(y-y_{j}\right)^{2}}}{C_{g}}
\end{aligned}
$$

where $t_{i, j}(x, y)$ is the time of flight of the GW that propagates with a group velocity $C_{g}$ from the transmitter $i$ located at $\left(x_{i}, y_{i}\right)$ to the inspected point $(x, y)$ and from the latter to the receiver $j$ at $\left(x_{j}, y_{j}\right)$. The group velocity $C_{g}$ can be determined either using various simulation frameworks, such as semi-analytical finite elements method [24], or it can be experimentally measured. Finally, each residual signal is delayed by this value, so that each point of the image can be associated with a part of the envelope, and the DI value is computed as follows:

$$
D I^{D A S}(x, y)=\sum_{i=1}^{N-1} \sum_{j=i+1}^{N} r_{i, j}\left(t_{i, j}(x, y)\right) .
$$

\subsubsection{Minimum Variance algorithm}

Hall et al. [15] proposed the Minimum Variance algorithm, which can be considered as an advanced version of DAS. It takes into account a diffraction pattern of GWs scattered by a defect in order to improve the resolution of the image. For each pixel, a vector of time-delayed signals is constructed as follows:

$$
\begin{aligned}
\vec{r}(x, y, t)=\left[\left|\tilde{r}_{1,2}\left(t+\frac{d_{1,2}(x, y)}{C_{g}}\right)\right|, \ldots\right. \\
\\
\left.\left|\tilde{r}_{N-1, N}\left(t+\frac{d_{N-1, N}(x, y)}{C_{g}}\right)\right|\right]^{T}
\end{aligned}
$$

where $\vec{r}(x, y, t)$ is the vector of time-delayed signals, $d_{i, j}(x, y)=\sqrt{\left(x-x_{i}\right)^{2}+\left(y-y_{i}\right)^{2}}+\sqrt{\left(x-x_{j}\right)^{2}+\left(y-y_{j}\right)^{2}}$ denotes the Euclidian distance to the pixel $(x, y)$ for the $i, j$ pair of transducers and $C_{g}$ is a group velocity of the guided mode. A correlation matrix, which is identical to the DAS DI mapping [25], is then computed for each pixel $(x, y)$ as follows:

$$
R(x, y)=\sum_{t=t_{1}}^{t_{2}} \vec{r}(x, y, t) \vec{r}^{\dagger}(x, y, t),
$$

where $\dagger$ denotes the complex conjugate, $t_{1}$ and $t_{2}$ are the temporal limits of the wave packet. A weighting vector $\vec{W}(x, y)$ is constructed in order to minimize false alarm that may be present in the original DAS map. Specifically, elements of this vector are selected to satisfy the following optimization problem:

$$
\vec{W}_{i, j}(x, y)=\operatorname{argmin}\left(\vec{w}_{i, j}^{\dagger} R_{i, j}(x, y) \overrightarrow{w_{i, j}}\right)
$$

with

$$
\vec{w}_{i, j}^{\dagger} \vec{e}_{i, j}(x, y)=1
$$

where $\vec{e}(x, y)$ is an unit vector, which describes the directional relation between the $\mathrm{GW}$ signals and $\vec{w}$ represents a 
weighting vector. In order to obtain $\vec{w}$, the unit vectors $\vec{e}(x, y)$ are computed as follows:

$$
\vec{e}_{i, j}(x, y)=\left[\frac{\phi_{1,2}}{\sqrt{d_{1,2}(x, y)}}, \ldots \frac{\phi_{N-1, N}}{\sqrt{d_{N-1, N}(x, y)}}\right]
$$

where the denominators represent the product of the distance traveled by the wavepacket while propagating from the emitter at $\left(x_{i}, y_{i}\right)$ to the observation point $(x, y)$ and to the receiver located at $\left(x_{j}, y_{j}\right)$. The corresponding numerators $\phi_{i, j}$ represent the scattering coefficients that characterize the amount of energy scattered by a defect at location $(x, y)$ for a given $(i, j)$ PZT pair. The optimal values for the $\phi_{i, j}$ depend on the shape of the defect and its orientation. Unless assumptions can be made regarding the type, shape and orientation of the defect, a common practice is to assume the defect is omnidirectional, that is $\phi_{i, j}=1$ for all pairs of transducers. The next step consists in minimizing the DI value to the defect free zone, while preserving DI values of the flawed zone. Finally, the DI is computed as follows:

$$
D I^{M V}(x, y)=\vec{W}_{i, j}(x, y)^{\dagger} R(x, y) \vec{W}_{i, j}(x, y) .
$$

\subsubsection{Excitelet algorithm}

GWs dispersion phenomenon introduces additional complexity to the defect imaging procedure [26, 27], and was neglected in both DAS and MV algorithms. Quaegebeur et al. [16] have proposed a correlation-based imaging algorithm, named Excitelet, where the damage is modeled as a perfect omnidirectional reflector. For each pixel $(x, y)$, it calculates a normalized coefficient of correlation between the residual signal and the analytically propagated signal corresponding to the same path. More specifically, the DI value is computed as follows:

$$
D I^{E X C}(x, y)=\sum_{i=1}^{N-1} \sum_{j=i+1}^{N}\left|\frac{\operatorname{cov}\left(u_{i, j}(t), s_{i, j}(x, y, t)\right)}{\sigma\left(u_{i, j}(t)\right) \sigma\left(s_{i, j}(x, y, t)\right)}\right|,
$$

where cov denotes covariance, $\sigma$ is a standard deviation, $u_{i, j}(t)$ is a residual experimental signal and $s_{i, j}(x, y, t)$ denotes an analytical signal that describes the GW traveling from the emitter to the receiver via the observation point.

The analytical signal can be expressed using the Green function formalism in the frequency domain for a given frequency range. The Fourier transform of the Green function can be expressed in terms of a real-valued spatial frequency. It consists of homogeneous and inhomogeneous components, where both parts are necessary to describe an out-going wave satisfying causality. For isotropic plate-like structures, it can be approximated as follows:

$$
\begin{array}{r}
s_{i, j}(x, y, t)=\left(\int_{-\infty}^{\infty}-\frac{2 j \Gamma_{m}(\omega)}{\pi} \frac{\exp \left(-j k_{m}(\omega) r_{1}\right)}{\sqrt{k_{m}(\omega) r_{1}}}\right. \\
\left.\frac{\exp \left(-j k_{m}(\omega) r_{2}\right)}{\sqrt{k_{m}(\omega) r_{2}}} \exp (-j \omega t) d \omega\right) * f_{\text {exc }}(t)
\end{array}
$$

where $\Gamma_{m}$ is the transducer frequency response, $k_{m}(\omega)$ is the wavenumber of the guided mode under consideration, $r_{1}$ and $r_{2}$ are the distances from the emitting transducer to the point of observation and from the latter to the receiving transducer, respectively and $f_{\text {exc }}(t)$ is the excitation function.

\section{Database generation}

The GWI algorithms are applied to an extensive database of simulated GWs signals in order to evaluate their localization performance under perfect and varying environmental conditions. The database is generated by means of SHM package of CIVA software to simulate the GW propagation in aluminum plates with randomly distributed defect size and location. GWs signal are then deteriorated using linear model for GWs modification due to environmental effects.

\subsection{GWs simulation framework}

The GWs simulations using Finite Element (FE) methods are usually time consuming due to the small element size and time step requirements [28]. A recent benchmark [29] compares the efficiency of four different FE software, namely in-house EFIT code, Abaqus, Comsol and Ansys, on a specific use case, which represents the GWs propagation in a carbon fiber reinforced polymer plate from a piezoelectric transducer over a delaminated region. The performance results of this benchmark are presented in terms of computational time required for this single simulation, which varies from 20 to 90 hours, with memory usage from tens of GB to hundreds of GB. Such performance makes the generation of an extensive database for statistical studies impossible.

In the present work, CIVA is used to generate an extensive database of GWI samples. It relies on the full threedimensional Spectral Finite Element (SFE) solution of the elastodynamic equations [30]. The SFE code uses two main concepts to speed up the simulation time. First, a high order spectral finite element method is implemented to significantly reduce the number of elements [31]. Secondly, a macro-element pre-meshing strategy is implemented to optimize global mesh considering its identical regions and an implicit element orientation. This leads to the significant reduction of CPU usage and memory footprint. The simulation configuration used in the benchmark [29] was reproduced with a computational time of about 10 minutes on a regular desktop computer and a memory usage of about 100 MB for quantitatively identical results [32]. The reduction of both memory load and computational time allows running 
multiple simulations either in parallel or sequentially and enable the creation of an extensive database of simulated GWI results in a reasonable amount of time.

\subsection{GWs signal degradation model}

Environmental effects on GWs propagation are primarily pronounced in modal amplitude modification as well as changes in group and phase velocities. They are conditioned by various thermal effects, including material expansion and change in stiffness as well as change in piezoelectricity of transducers and their bonding [33]. It has been demonstrated that GWs signals can be numerically manipulated with respect to these phenomena in order to mimic variations in environmental effects and operational conditions as follows [34]:

$$
S(t)_{d}=\alpha S(t(1+\beta))+N(0, \sigma),
$$

where $\alpha$ represents amplitude variation, $\beta$ GWs signal stretching due to thermal effects and $N(0, \sigma)$ represents additive white Gaussian noise. On the basis of experimental results, all the three degradation parameters are supposed to vary in following ranges complying with random uniform distributions: $\alpha \subset[0.875,1.125], \beta \subset[-0.0005,0.0005]$ and $\sigma \subset[0,0.02]$.

They were selected to approximately cover a degradation range of GWI results that were observed experimentally while performing defect imaging under varying temperature with $\left|d T_{\max }\right| \approx 20^{\circ} \mathrm{C}$. More details on the calibration of the GWs degradation model can be found in the following work [35].

\subsection{Database description}

The studied configuration is an aluminum plate $600 \mathrm{~mm}$ x $600 \mathrm{~mm}$ x $3 \mathrm{~mm}$, which is instrumented with eight identical circular PZT transducers $18 \mathrm{~mm}$ in diameter. In this simulation, it is assumed that the excitation is purely radial because of the low-frequency range [36]. The piezoelectric load is modeled as a axisymmetric in-plane radial force applied to the surface of the plate on the outer perimeter of the sensor [37]. The same model is used for the receivers, for which the radial strain is integrated over the perimeter of the sensors. In total, 500 simulations were run for three inspecting frequencies.

Each simulated sample contains a through-hole of varying size and position. Holes are distributed within the circle of $100 \mathrm{~mm}$ in radius with the origin at the center of the plate. The distribution of the defects is represented by the red circles in Figure 2a. This configuration ensures that the distance between defects and PZTs of at least one wavelength of the inspecting mode. The damage radii vary from $2.5 \mathrm{~mm}$ to $7.5 \mathrm{~mm}$ corresponding to the typical defect size sought in aerospace applications and comply with a random uniform distribution.

The aluminum plate is inspected with two-cycle Hanning modulated bursts. Excitation central frequencies are set to the typical frequency range of use, namely $20 \mathrm{kHz}, 40 \mathrm{kHz}$ and $60 \mathrm{kHz}$, below the first cut-off frequency, in order to generate only two fundamental guided modes $A_{0}$ and $S_{0}$ and to optimize GWs-defect interaction.

The $A_{0}$ mode is selected for imaging for two main reasons. Firstly, the corresponding wavelength is smaller than that of the $S_{0}$ mode, which results in enhanced GWI resolution. Secondly, in the studied frequency range, the amplitude of $A_{0}$ mode measured by the transducer is higher than that of $S_{0}$ mode. This is shown by the excitability curves in Figure 2b computed using the framework proposed in [37]. Because the same transducer model is used for both emitters and receivers, the excitability curve is applied at both the emission and reception stages of the guided waves by the sensors. . The wavelengths associated with the excitation center frequencies of the $A_{0}$ mode are the following: $\lambda_{A_{0}}^{20 \mathrm{kHz}}$ $=37.9 \mathrm{~mm}, \lambda_{A_{0}}^{40 \mathrm{kHz}}=26.24 \mathrm{~mm}$ and $\lambda_{A_{0}}^{60 \mathrm{kHz}}=21.19 \mathrm{~mm}$, so that the GWs-defect interaction is optimized. Note that the defect sizes under consideration in this paper are rather large, which leads to satisfying detections even with low inspection frequencies. This choice is made to ensure direct compatibility of the study in this paper with composite inspection with large impact defects and high attenuation. Moreover, it is assumed that a single defect exists within the inspection area, and all the coupled interactions between multiple nearby defects are neglected.

Each database sample is processed with the three previously described algorithms. For illustrative purposes, images obtained with DAS, MV and Excitelet under ideal environmental conditions are presented in Figure 3. Their resolution is directly related to the wavelength of the propagating guided mode: an increase of wavelength leads to the decrease of the resolution capability of the imaging algorithms. Intuitively, inspecting a structure at higher frequencies should lead to better defect localization results. However, it is not necessarily true, and it will be demonstrated that for a given GWI configuration and a range of defect sizes, an optimal inspection frequency can be identified.

\section{Analysis}

In this section, GWI results obtained with DAS, MV and Excitelet algorithms are discussed. Predicted defect coordinates are compared to their reference values, and localization errors as well as corresponding probabilities are evaluated for both cases: perfect operational conditions and the ones comprising environmental changes. From this analysis, the best combination of imaging algorithm and inspection frequency are determined.

\subsection{GWI results observation}

It was demonstrated that the DI spatial distribution complies with a Gaussian distribution over the damage position [38]. Considering that there is only one defect per simulated dataset instance, the corresponding defect location is obtained by searching the location of maximum in the image. 


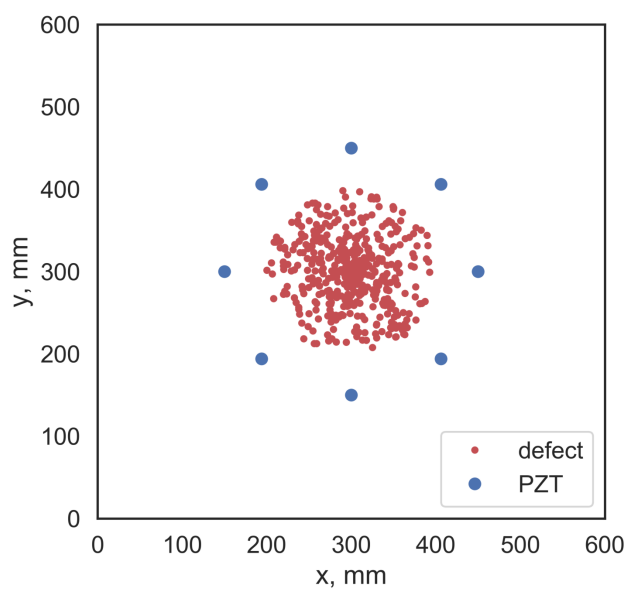

(a) GWI configuration.

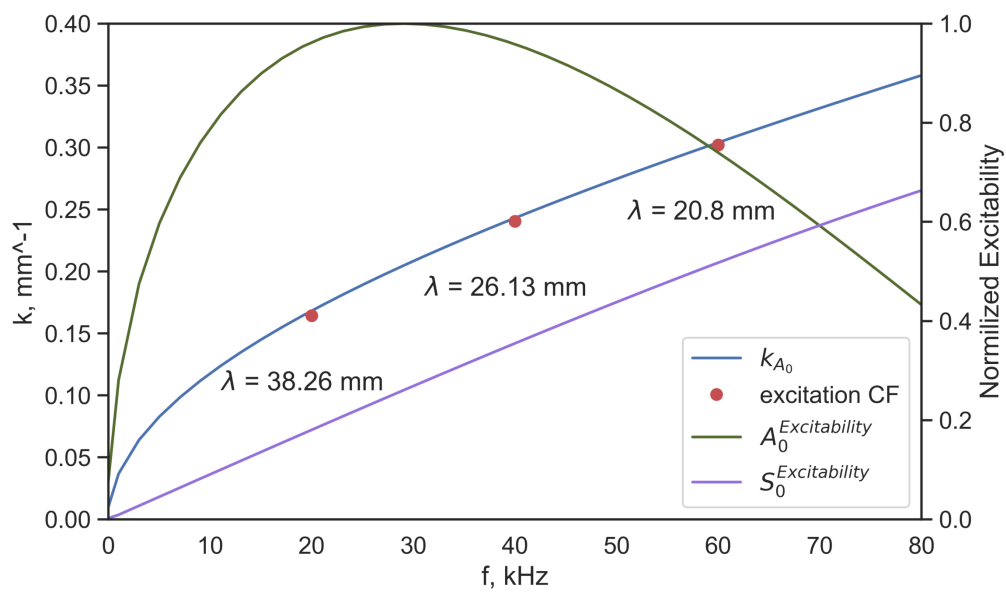

(b) The $A_{0}$ guided mode dispersion curve and wavelengths corresponding to excitation frequencies. Green and purples curves represent normalized excitability of $A_{0}$ and $S_{0}$ modes, respectively.

Fig. 2: Schematic representation of simulated database configuration.

Each family of observations that corresponds to the combination of imaging algorithm and excitation frequency under both perfect and varying operational conditions are shown in Figure 4. Observation are plotted against each other and their reference values in order to estimate the defect localization accuracy.

For both perfect and varying operational conditions at $20 \mathrm{kHz}$, all the three algorithms suffer from considerable localization errors due to the large wavelength of the inspecting mode $\left(\lambda_{A_{0}}^{20 k \mathrm{~Hz}}=37.9 \mathrm{~mm}\right)$ with respect to the transducers inter-distances. The largest distance between transducers approximately corresponds to four wavelengths of the inspecting mode, so the wave packets corresponding to $A_{0}$ and $S_{0}$ modes in the residual signal are heavily overlapped. Among the three algorithms, Excitelet (represented by green triangles) demonstrates the best coherence between observed defect coordinates and their true values. For this algorithm operating under perfect conditions, there is a systematic localization underestimation for the defects remote more than 20 $\mathrm{mm}$ from the center of the plate. We have not identified the origin of this phenomenon. However, when environmental modifications appear, a new systematic erroneous trend can be observed, see Figure 4 (c, d). It corresponds to extreme GWI cases where the damage is close to sensor's circumference and environmental variations are high.

Although the wave packets are overlapped, Excitelet is more prone to correctly map the residual signal than DAS and $\mathrm{MV}$, under perfect operational conditions as it computes a linear association between the residual and analytical signals. Such a linear association allows capturing differences in phase velocities of guided modes, while DAS and MV ignore them by mapping the envelope of the residual signal. However. when operation conditions are no longer perfect, DAS demonstrates its superiority.

As shown in Figure $4(\mathrm{e}-\mathrm{h})$, the inspection at $40 \mathrm{kHz}$ leads to more accurate localization results for all the three imaging algorithms for both perfect and varying operational conditions. Overall, Excitelet demonstrates the best statistical fit and the smallest variance for both predicted coordinates regarding their reference values. However, observations located beyond $80 \mathrm{~mm}$ from the center of the plate are progressively underestimated by Excitelet regardless operational conditions. As in the previous case, erroneous systematic trend can be observed. Perhaps, it can be analytically related to parameters of the GWs signals degradation model, but we did not managed to identify it explicitly. It will be demonstrated below that the probability of this event is extremely low and decreases with frequency so that it does not disturb overall statistical performance of the GWI algorithm.

The analysis of GWI results at $60 \mathrm{kHz}$ reveals that the defect localization performance starts degrading for all the three imaging algorithms under both perfect and varying operational conditions. At this frequency, the $A_{0}$ mode excitability decreases in comparison with that at $20 \mathrm{kHz}$ and 40 $k H z$, see Figure $2 \mathrm{~b}$. Consequently, residual signal contains more of the $S_{0}$ mode obscuring the $A_{0}$ mode component that should be mapped by defect imaging algorithms. This adversely interfere in DAS and MV computations as they map residual energy. A part of the residual signal, which corresponds to the defect-free location, contains wave packets that are not taken into account by a single mode imaging procedure. On the contrary, Excitelet is less affected by this phenomenon as it relies on the normalized correlation coefficient computation between analytically propagated $A_{0}$ mode and experimental residual signal. Obviously, the normalization term in Eq. (10) increases due to additional harmonics in the residual signal resulting in the decrease of the DI value but the rate of change is less significant in comparison with DAS and MV. Hence, Excitelet still demonstrates the best performance, but its variance has grown in comparison with $40 \mathrm{kHz}$. It should be mentioned that when operational conditions vary, all the three GWI algorithm suffer less from the 

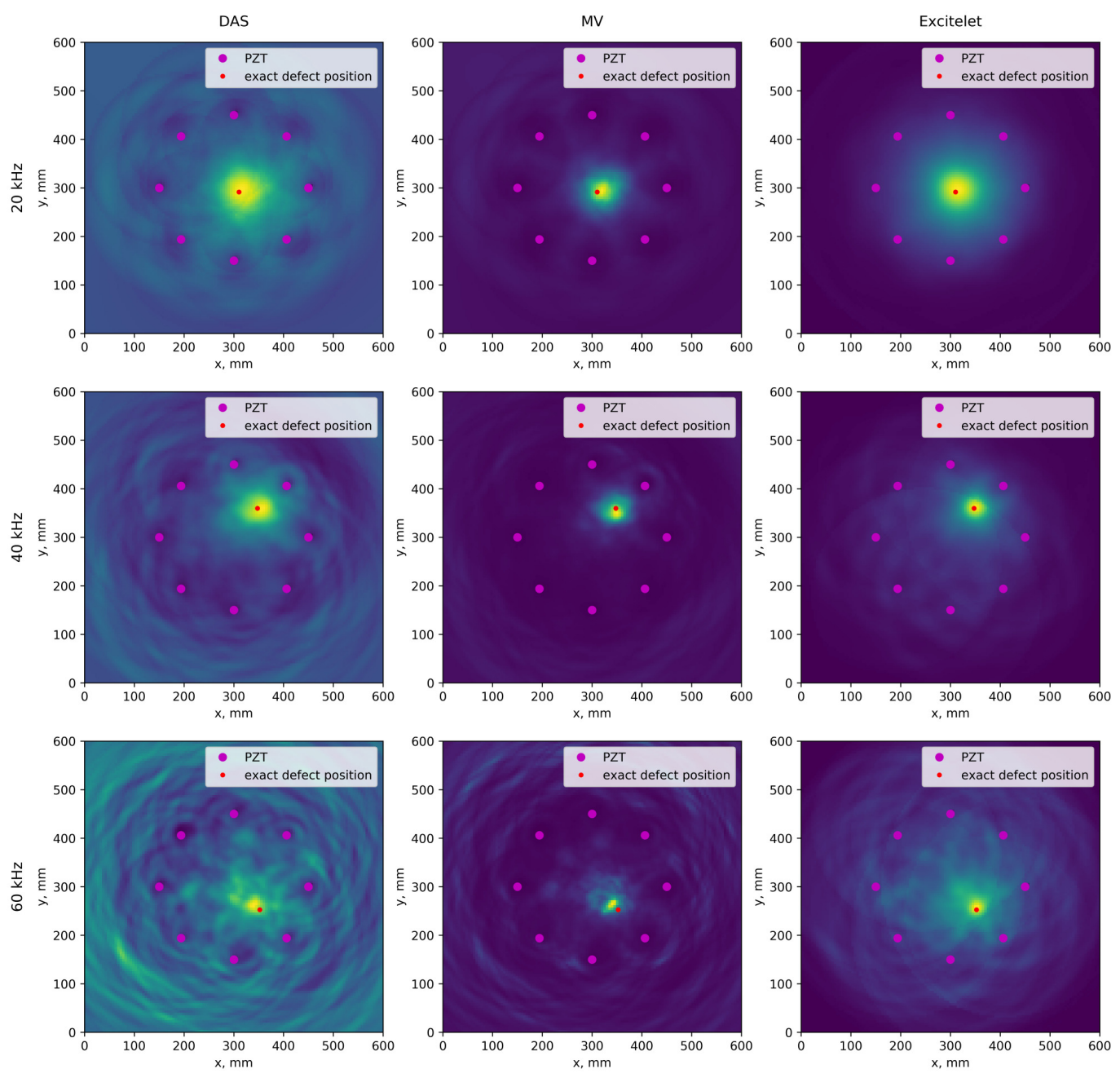

Fig. 3: Defect maps computed with DAS (left column), MV (middle column), and Excitelet (right column) with inspection central frequency of $20 \mathrm{kHz}$ (top line), $40 \mathrm{kHz}$ (mid line) and $60 \mathrm{kHz}$ (bottom line) under perfect environmental conditions.

aforementioned systematic errors (further investigations are required to understand the nature of it).

These observations, made on the fairly simple GWI configuration, allows revealing inherent weaknesses of imaging algorithms that can deteriorate imaging performance under perfect/varying operational conditions. It is supposed that they can be translated to realistic applications where the GWs propagation and interaction with structural features are more complex.

\subsection{Statistical localization errors}

Absolute Localization Error (ALE) is obtained by computing the $L_{1}$ norm between true and observed defect locations. While a single ALE does not provide sufficient insight into GWI algorithm performance, its Probability Density Function (PDF) on the possible sample space can reveal a likely outcome of the GWI process. Hence, statistical inference about ALE probability distribution regarding the defect size can be deduced from their joint occurrence.

Therefore, ALE are collected over the whole database of GWI results, and PDFs are computed for all families of localization errors and frequencies using the Kernel Density Estimate (KDE) method relying on the radial basis function kernel as follows:

$$
\hat{p}_{n}(x)=\frac{1}{n h} \sum_{i=1}^{n} K\left(\frac{X_{i}-x}{h}\right),
$$

where $K(x)$ is a smooth and symmetric kernel function, $h>$ 0 is the smoothing bandwidth, and $X_{i}$ represents the data. Basically, the KDE smooths each data point $X_{i}$ into a small density bumps and then sum all these small bumps together in order to obtain the final density estimate. For more details on KDE, the reader is invited to refer to [39]. This method is chosen as it yields an empirical estimate of the PDF without assuming any form of underlying density function.

The ALE PDF depends on the defect size as GWsdamage interaction is conditioned by the $\lambda / \oslash$ ratio and reaches it's maximum when $\lambda / 2=\oslash$. For all the three imaging central frequencies, ALE bivariate PDFs under perfect and varying operational conditions are plotted against defect sizes, see Figure 5 and Figure 6, respectively. 

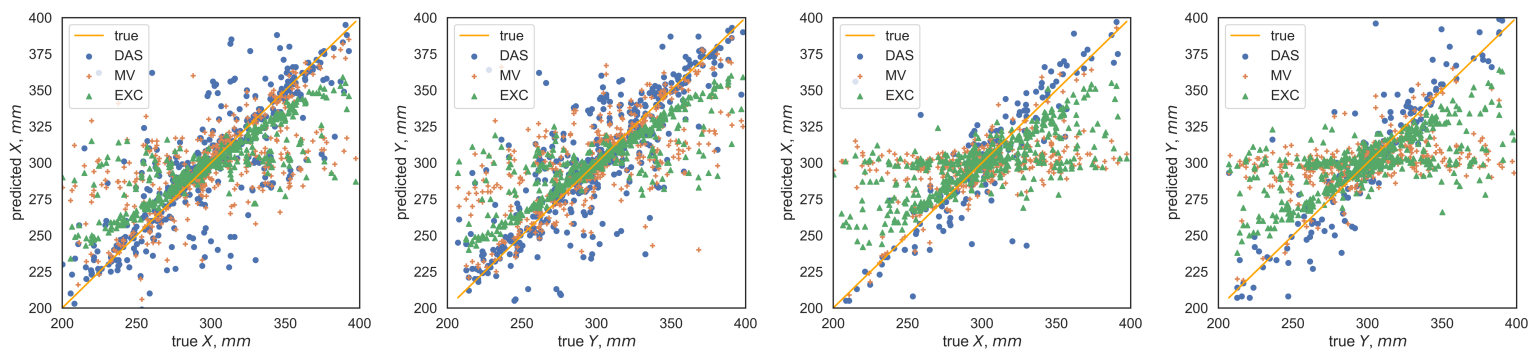

(a) $X$ coordinate predictions at (b) $Y$ coordinate predictions at (c) $X$ coordinate predictions at (d) $Y$ coordinate predictions at $20 \mathrm{kHz}$.

$20 \mathrm{kHz}$.

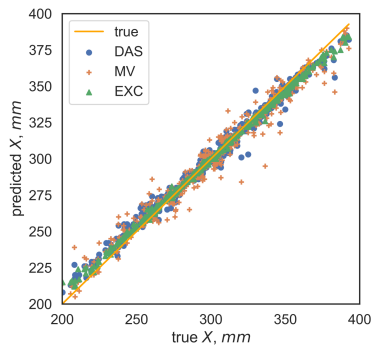

(e) Defect $X$ coordinate predictions at $40 \mathrm{kHz}$.

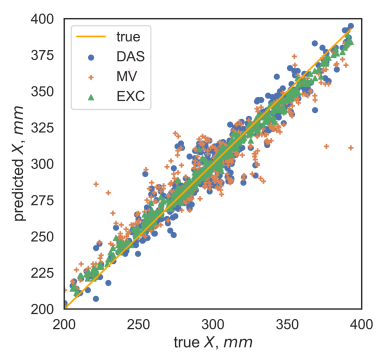

(i) Defect $X$ coordinate predictions at $60 \mathrm{kHz}$.

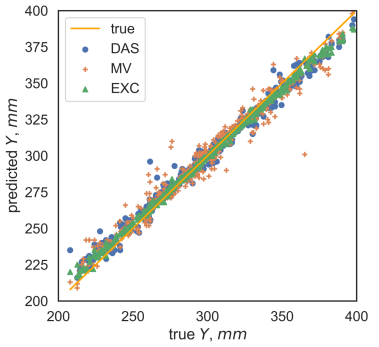

(f) Defect $Y$ coordinate predictions at $40 \mathrm{kHz}$.

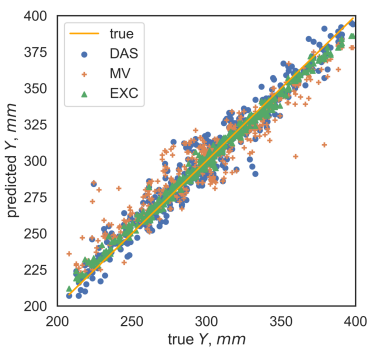

(j) Defect $Y$ coordinate predictions at $60 \mathrm{kHz}$.
$20 \mathrm{kHz}$.

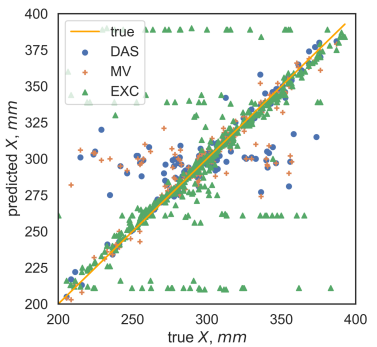

(g) Defect $X$ coordinate predictions at $40 \mathrm{kHz}$

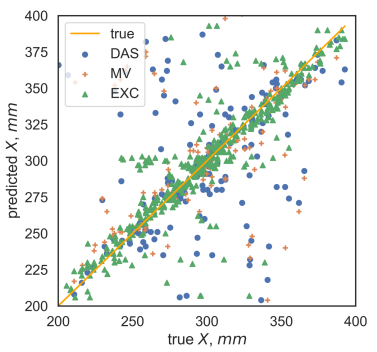

(k) Defect $X$ coordinate predictions at $60 \mathrm{kHz}$.

\section{$20 \mathrm{kHz}$.}

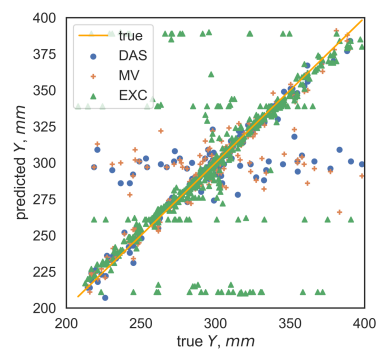

(h) Defect $Y$ coordinate predictions at $40 \mathrm{kHz}$

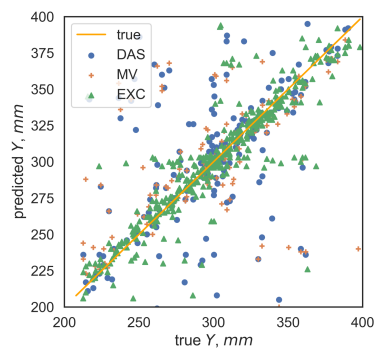

(1) Defect $Y$ coordinate predictions at $60 \mathrm{kHz}$.

Fig. 4: Defect location predictions under both perfect and varying operational conditions. The columns represent the defect localization results without (two columns on the left) and with (two columns on the right) varying operational conditions. Imaging results are obtained at $20 \mathrm{kHz}, 40 \mathrm{kHz}$, and $60 \mathrm{kHz}$ and are shown in this order from the top to the bottom row. The orange crosses, blue dots and green triangles represent the results obtained by DAS, MV and Excitelet respectively, while the yellow line denotes true values.

Under perfect conditions at $20 \mathrm{kHz}$, all the algorithms are less accurate in locating small defects. The ALE can reach $75 \mathrm{~mm}, 100 \mathrm{~mm}$ and $100 \mathrm{~mm}$ for DAS, MV and Excitelet while the wavelength of the inspecting mode is of about $38 \mathrm{~mm}$. At $40 \mathrm{kHz}$, localization errors are less significant. This frequency can be considered as optimal to search for defects in a given GWI configuration. The most frequent ALE is $7 \mathrm{~mm}, 5 \mathrm{~mm}$, and $3 \mathrm{~mm}$ for DAS, MV, and Excitelet algorithms, respectively. Excitelet demonstrates the most frequent absolute localization error of about $3 \mathrm{~mm}$. Moreover, the probability of ALE higher than $10 \mathrm{~mm}$ is extremely small, while the defect size varies from $5 \mathrm{~mm}$ to $15 \mathrm{~mm}$. At the same frequency, DAS and MV demonstrate the most probable error of about $8 \mathrm{~mm}$ because they do not account the GWs dispersion phenomena. Additionally, MV occasionally suffers from large localization errors due to the assumption of omnidirectional defect diffraction pattern. When inspection frequency is $60 \mathrm{kHz}$ the ALE PDFs increase for the three GWI algorithms due to decrease in $A_{0}$ excitability as previously discussed.

In practice, a probability of experiencing ALE larger than maximum tolerable error is required. In the present study, the maximum tolerable error is arbitrarily fixed at $15 \mathrm{~mm}$ corresponding to the largest transverse hole, and the probability $P$ of having ALE larger than maximum tolerable error $P(A L E>15, \mathrm{~mm})$ can be evaluated by integrating the PDF between the corresponding bounds. Other maximum tolerable error could be defined depending on the application. At $20 \mathrm{kHz}$ the $P(A L E>15, \mathrm{~mm})$ are quite high and equal $56.2 \%, 70.2 \%$ and $61.7 \%$ for DAS, MV and EXC algorithms. Consequently, GWI results obtained at this frequency cannot be considered as reliable. On the contrary, 

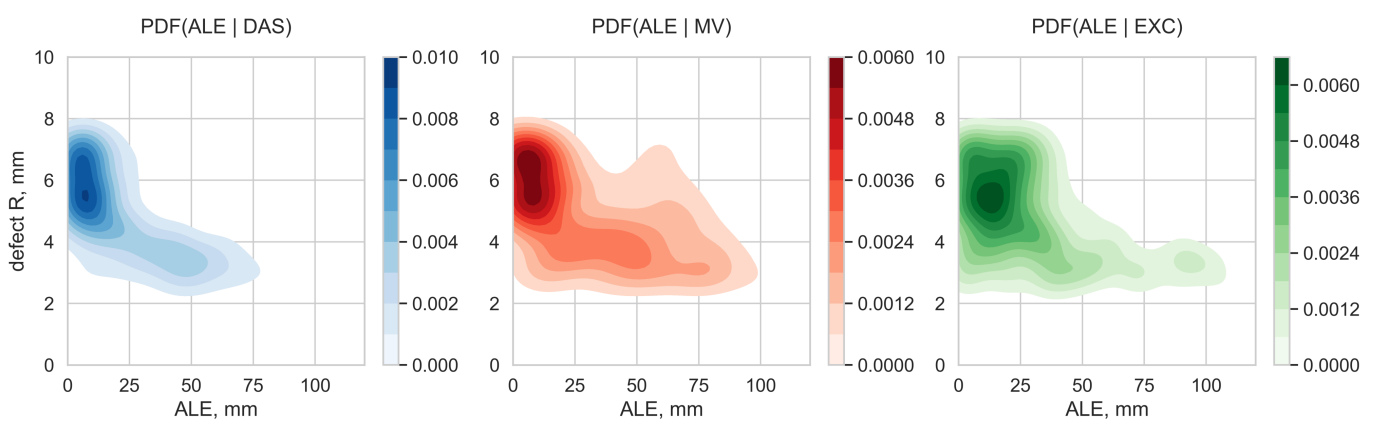

(a) PDFs of GWI algorithms ALE versus defect size under perfect operational conditions at $20 \mathrm{kHz}$.
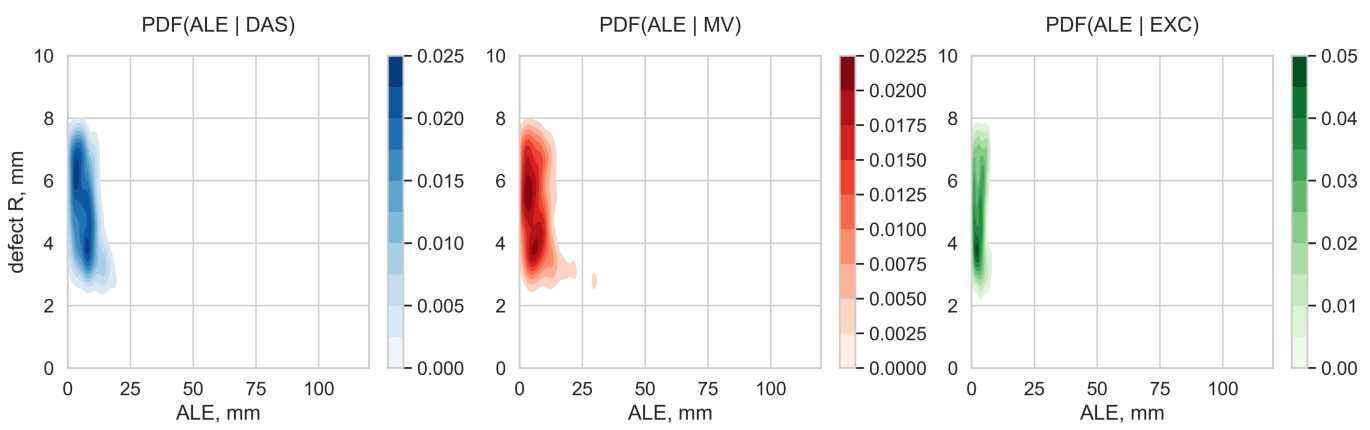

(b) PDFs of GWI algorithms ALE versus defect size under perfect operational conditions at $40 \mathrm{kHz}$.
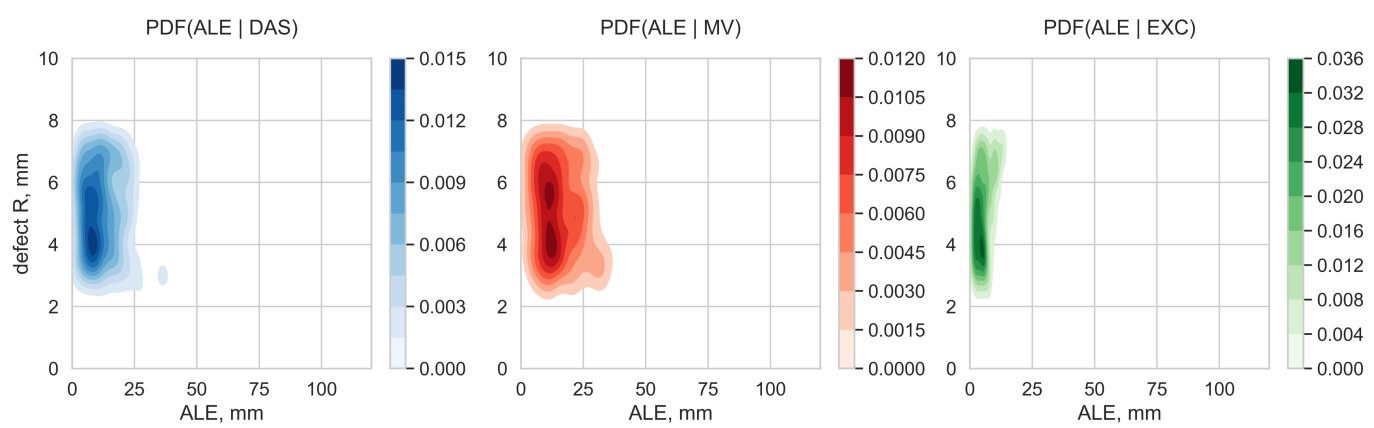

(c) PDFs of GWI algorithms ALE versus defect size under perfect operational conditions at $60 \mathrm{kHz}$.

Fig. 5: Defect location predictions under perfect operational conditions. Observations and reference values of $X$ and $Y$ are plotted in left and right columns respectively. The imaging results are obtained at $20 \mathrm{kHz}, 40 \mathrm{kHz}$, and $60 \mathrm{kHz}$ and are shown in this order from the top to the bottom row. The orange crosses, blue dots and green triangles represent the results obtained by DAS, MV and Excitelet respectively.

at $40 \mathrm{kHz}$ the corresponding results are $9.9 \%, 21.4 \%$ and $0.15 \%$, and at $60 \mathrm{kHz} P(A L E>15, \mathrm{~mm})$ are $44.3 \%, 57.0 \%$ and $1.0 \%$, respectively. Note that a $P(A L E>15, \mathrm{~mm})=1 \%$ means that the probability of an accurate localization is $99 \%$. It is worth noting the remarkable performance of Excitelet algorithm in comparison to the two other algorithms, while the structure is inspected at these frequencies.

Even small environmental variations significantly decrease performance of GWI algorithms so that PDFs become spread, see Figure 6. Previously mentioned weaknesses are amplified due to amplitude variation, phase/group velocities shifts and measurement noise. The ALE can reach and overcome $100 \mathrm{~mm}$ for all the three GWI algorithm at $20 \mathrm{kHz}$, and the GWI results are generally poor: $P(A L E>15, \mathrm{~mm})$ are high and equal $80.71 \%, 77.54 \%$ and $70.09 \%$ for DAS, MV and EXC algorithms, respectively.

At $40 \mathrm{kHz}$, GWI results are more accurate, PDFs of localization errors are less significant so that the $P(A L E>$ $15, \mathrm{~mm})$ is the following for all the three algorithms: 54.23 $\%, 46.69 \%$ and $42.73 \%$. When inspection frequency is $60 \mathrm{kHz}$, GWI results start degrading as under perfect operational conditions, and ALE PDFs are spreading out so that the $P(A L E>15, \mathrm{~mm})$ are $68.22 \%, 67.64 \%$ and $46.31 \%$ for DAS, MV and Excitelet algorithms.

\subsection{Performance metrics}

General practice in model verification is to determine the alignment of model predictions with the reference values. It typically involves computing a set of performance metrics in order to capture all aspects of the model behavior, 


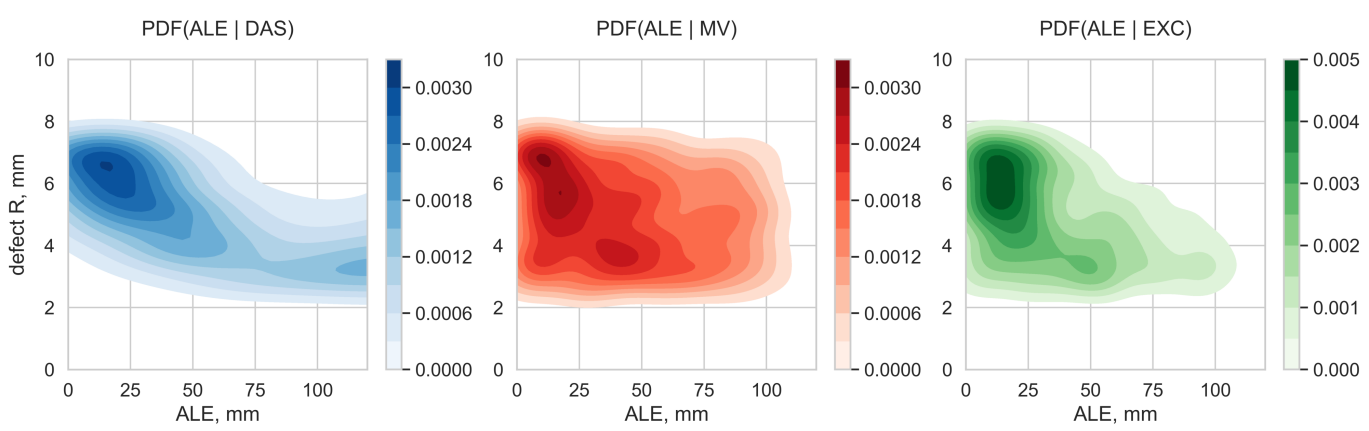

(a) PDFs of GWI algorithms ALE versus defect size under varying operational conditions at $20 \mathrm{kHz}$.

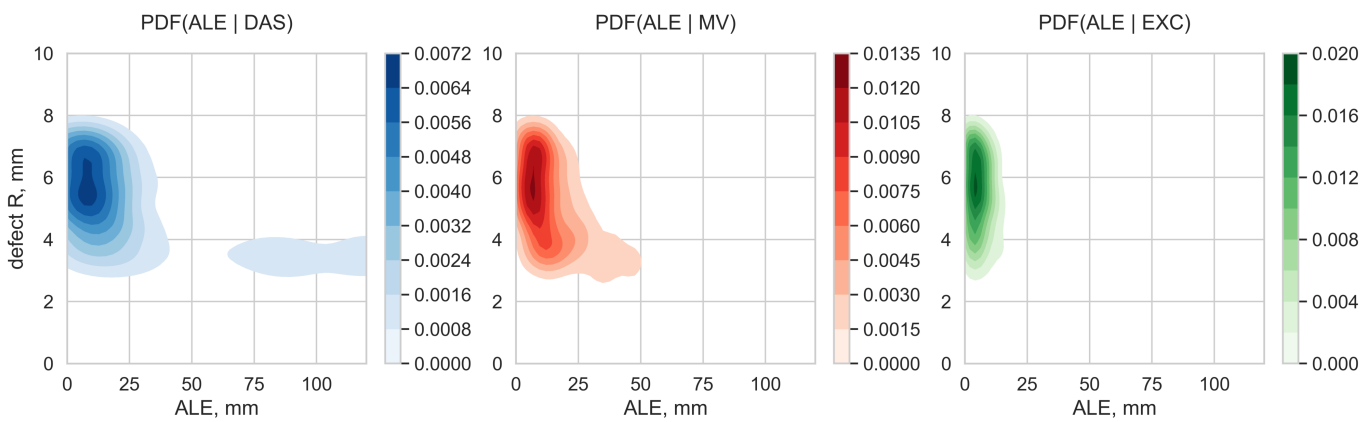

(b) PDFs of GWI algorithms ALE versus defect size under varying operational conditions at $40 \mathrm{kHz}$.

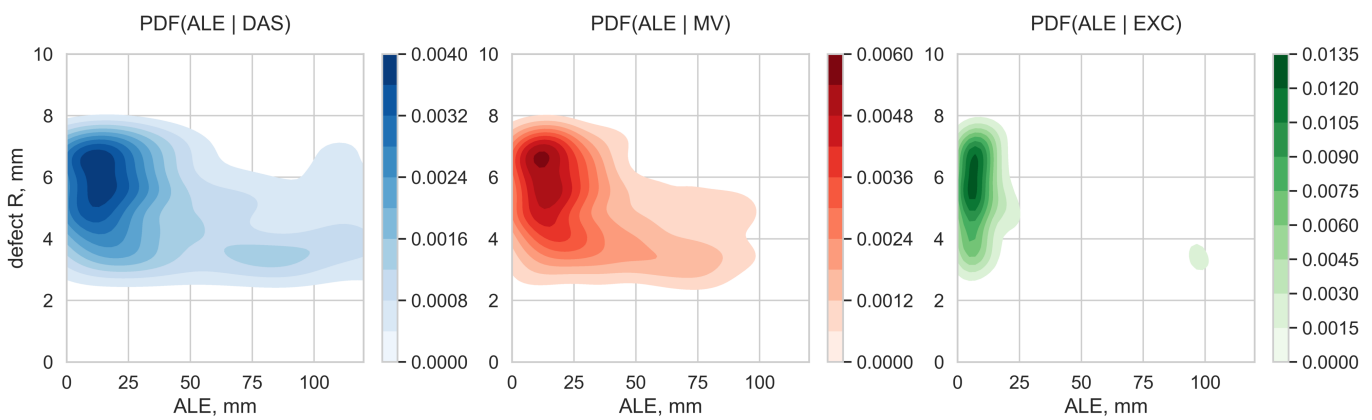

(c) PDFs of GWI algorithms ALE versus defect size under varying operational conditions at $60 \mathrm{kHz}$.

Fig. 6: Defect location predictions under perfect operational conditions. Observations and reference values of $X$ and $Y$ are plotted in left and right columns respectively. The imaging results are obtained at $20 \mathrm{kHz}, 40 \mathrm{kHz}$, and $60 \mathrm{kHz}$ and are shown in this order from the top to the bottom row. The orange crosses, blue dots and green triangles represent the results obtained by DAS, MV and Excitelet respectively.

reflecting statistical similarities and differences between true values and experimental observations. For more details on the computation of the error metrics, the reader is invite to refer to [40].

Mean Absolute Error (MAE) measures the average magnitude of errors over the dataset without considering their directions. In our case, it is computed as an average of the absolute differences between the maxima of images and the corresponding reference coordinates, where all individual differences have equal weight. A Root Mean Square Error (RMSE) represents a quadratic mean of model deviations from reference values. Each RMSE component is proportional to squared error which makes this metric highly sensitive to the presence of significant deviations, emphasizes the inability of the imaging algorithm to compute an image correctly. The Standard Deviation (STD) is a statistic that evaluates a dataset dispersion. STD of model errors provides insights on the incertitude experienced by the imaging algorithm. For example, the spread of the predictions at $20 \mathrm{kHz}$, shown in Figure 4, generally happens when defect imaging algorithms fail to reconstruct images. Finally, a product-momentum Coefficient of Correlation $(\mathrm{CoC})$ is used to determine the strength of the linear statistical relationship between observations and their reference values, where the perfect alignment of both leads to $C o C=1$ and the absence of linear relationship results in $C o C=0$.

The performance metrics of all the three defect imaging algorithms are collected under perfect and varying operational conditions and summarized in Table (1) in order to estimate a degree of correspondence between the imaging al- 
Table 1: Performance metrics summery for Excitelet, DAS and MV defect imaging algorithms under perfect operational conditions (POC) and varying operational conditions (VOC). Results for $20 \mathrm{kHz}, 40 \mathrm{kHz}$ and $60 \mathrm{kHz}$ are grouped central inspection frequency.

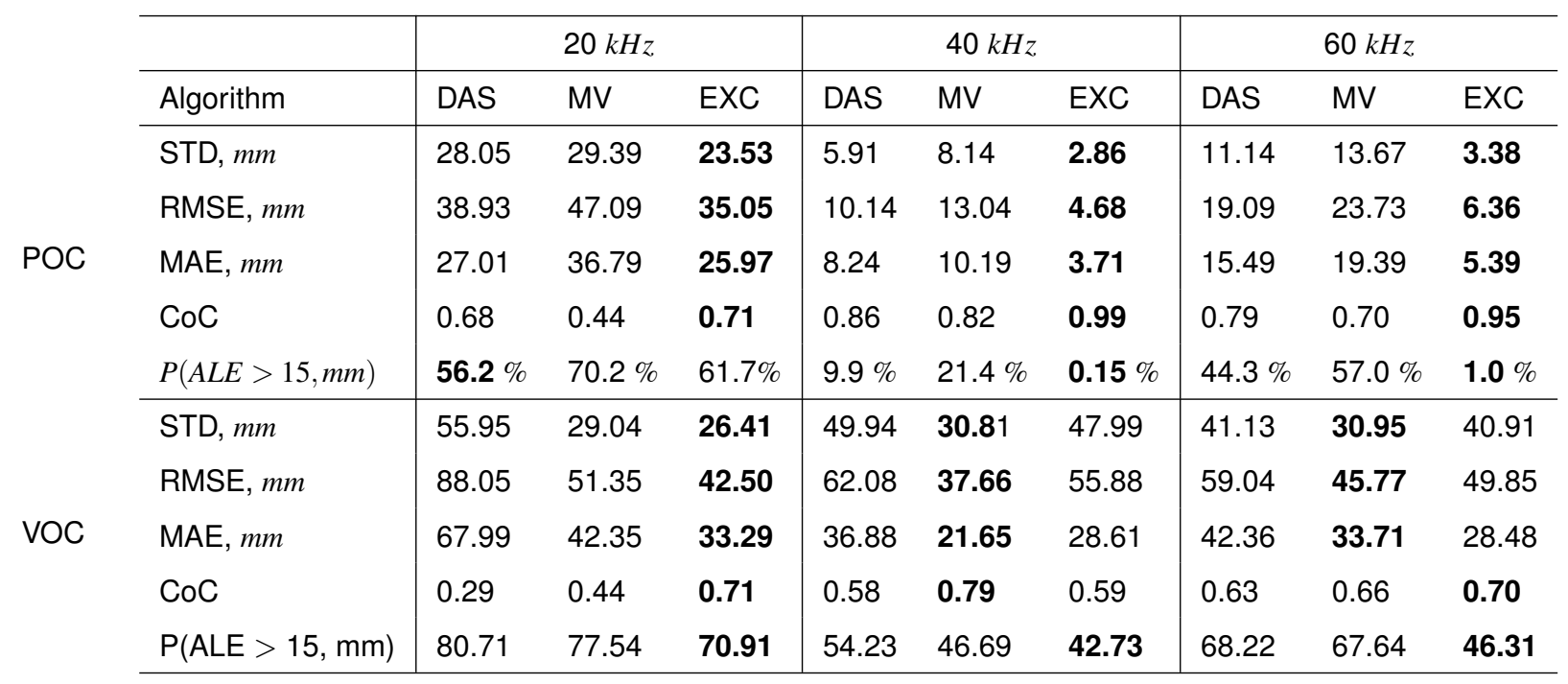

gorithms outputs and the reference values. In this table, the STD, RMSE, MAE, and CoC are grouped by excitation frequencies for all the three imaging algorithms, so the merits of competing imaging algorithms and excitation frequencies can be determined for a given GWI configuration. From the analysis of Table (1), it can be deduced that under perfect operational conditions the best defect localization accuracy is achieved when the structure is monitored at $40 \mathrm{kHz}$ and imaged with Excitelet algorithm. Such a GWs imaging configuration allows obtaining a $C o C_{E X C}^{40 \mathrm{~Hz}} \approx 0.99$ predictions, while the RMSE and MAE are approximately equal to 4.86 $\mathrm{mm}$ and $3.71 \mathrm{~mm}$, respectively. Such low and close by magnitude RMSE and MAE errors indicate that Excitelet algorithm does not fail in image reconstruction. The STD, which indicates how the data is distributed around the mean value, estimates model's uncertainty with respect to the reference data. For all the three excitation frequencies, Excitelet is the least uncertain, and MV is the most volatile in localizing the defects.

Excitelet shows the best performances in terms of RMSE, MAE and MAE localization error metrics for all the three studied frequencies and reaches its peak while monitoring the structure at $40 \mathrm{kHz}$. The same conclusion holds for the $\mathrm{CoC}$ while measuring linear model alignment with reference values and searching for the best prediction accuracy.

However, when GWI is performed under varying operation conditions, significant decrease in algorithms performance is observed. Metrics demonstrate that environment modifications impact the most higher frequency damage imaging. The best GWI result at $20 \mathrm{kHz}$ is achieved by Excitelet algorithm where the STD is equal to $26.41 \mathrm{~mm}$, MAE is $33.29 \mathrm{~mm}$ and $\mathrm{CoC}$ is 70.91 . When frequency increases to $40 \mathrm{kHz}$, mean metrics demonstrate that the localization accuracy is significantly decreased for all the three GWI algorithms and the best result is achieved by MV algorithm where STD is $30.81 \mathrm{~mm}$ and MAE equals to 21.65 $\mathrm{mm}$. The same tendency is observed at $60 \mathrm{kHz}$, where MV outperforms its counterparts where STD equals to $30.65 \mathrm{~mm}$ and MAE is $33.71 \mathrm{~mm}$. However, for all the three algorithms $\mathrm{CoC}$ is always higher while GWI is performed by Excitelet and corresponding ALE distributions are narrower, $P(A L E>$ $15, \mathrm{~mm})$ is greater than for two other algorithms meaning that aforementioned metrics are biases by occasional high localization errors where algorithms failed to compute a correct image. Rather, a number of incoherent peaks are observed and image is barely interpretative. Therefore, PDFs is more suitable for inferring statistical performance of GWI algorithms. For example, for all the three GWI algorithms, Excitelet demonstrates the lowest $P(A L E>15, \mathrm{~mm})$ which is equal to $70.91 \%, 42.73 \%$ and $46.31 \%$ at $20 \mathrm{kHz}, 40 \mathrm{kHz}$ $60 \mathrm{kHz}$ frequencies, respectively.

\section{Conclusions}

This paper presents statistical studies on the localization performance of three state-of-art defect imaging algorithms for a GW-based SHM system under perfect and varying operational conditions. The study is conducted on numerically generated database of GWI results using GWs signals degradation model. The GWI configuration is fairly simple where the complex phenomena of GWs propagation and GWs- defect and boundaries interaction are reduced as much as possible for the sake of revealing the driving parameters of localisation performance inherent to defect imaging algorithms so that corresponding inferences can be arguably translated to more complex GWI configurations while developing GWsbased SHM systems for realistic applications.

The statistical advantage of using the Excitelet algorithm in comparison with DAS and MV is demonstrated. Excitelet requires the comprehensive knowledge of the GWs propagation in the structure for the analytical signal computation, it provides smaller localization errors. The lower per- 
formances of DAS and MV are due to the neglection of GWs dispersion which has to be taken into consideration for accurate localization. The three algorithms suffer from the mode conversion phenomena, emphasizing the need to adequately choose the inspection frequency to guarantee the best performances. For this specific configuration, this study leads to the conclusion that the best defect localization performance is achieved when the structure is monitored at $40 \mathrm{kHz}$ and imaging is performed using Excitelet algorithm. One of the main reasons that the GWI results are better at $40 \mathrm{kHz}$ for this plate is the diameter of piezoelectric transducer. This is a driving factor influencing the modal excitability. For this configuration, it is chosen to favor the $A_{0}$ generation so that an accurate localization of the defect is obtained for $99 \%$ of the cases. However, localization accuracy significantly decreases when operational conditions starts to vary and, for instance, Excitelet provides accurate defect imaging with the probability of $53.96 \%$ while temperature varies in the $\left|d T_{\max }\right| \approx 20^{\circ} \mathrm{C}$ range. Such GWI conditions require application of environmental effects compensation techniques. Their statistical advantage will be studied in the future work.

More generally, the presented methodology can contribute to the SHM system optimization and reliability evaluation. It allows to quantify the performance gain, but it has to be repeated for each constituent and configuration. The ALE probability distributions can also contribute to the reliability and associated risks estimation; it can be determined by a variety of methods, including Monte Carlo simulations. For future work, authors also plan to investigate robustness of these algorithms while detecting structural damage in composite plates for various GWI configurations and to include it into the performance demonstration of SHM system as a whole.

\section{References}

[1] Su, Z., Ye, L., and Lu, Y., 2006. "Guided Lamb waves for identification of damage in composite structures: A review". Journal of Sound and Vibration, 295(3-5), pp. 753-780.

[2] Diamanti, K., and Soutis, C., 2010. "Structural health monitoring techniques for aircraft composite structures". Progress in Aerospace Sciences, 46(8), pp. 342352.

[3] Sohn, H., Farrar, C. R., Hunter, N. F., and Worden, K., 2001. "Structural health monitoring using statistical pattern recognition techniques". Journal of dynamic systems, measurement, and control, 123(4), pp. 706711.

[4] Giurgiutiu, V., 2014. Structural Health Monitoring with Piezoelectric Wafer Active Sensors. Elsevier Academic Press.

[5] Farrar, C. R., and Worden, K., 2012. Structural Health Monitoring: A Machine Learning Perspective. Wiley.

[6] Kulakovskyi, A., Chapuis, B., Mesnil, O., Bedreddine, N.-R., d'Almeida, O., and Lhémery, A., 2017. "Defect imaging on CFRP and honeycomb composite structures by guided waves generated and detected by a sparse
PZT array". In Proceedings of the 11th International Workshop on Structural Health Monitoring.

[7] Chapuis, B., Terrien, N., and Royer, D., 2010. "Excitation and focusing of Lamb waves in a multilayered anisotropic plate". Journal of the Acoustical Society of America, 127(198).

[8] Cawley, P., 2018. "Structural health monitoring: Closing the gap between research and industrial deployment". Structural Health Monitoring, 17(5), pp. 12251244.

[9] Michaels, J. E., and Michaels, T. E., 2007. "Guided wave signal processing and image fusion for in situ damage localization in plates". Wave Motion, 44(6), pp. 482-492.

[10] Su, Z., Cheng, L., Wang, X., Yu, L., and Zhou, C., 2009. "Predicting delamination of composite laminates using an imaging approach". Smart Materials and Structures, 18(7), p. 074002.

[11] Qiu, L., Yuan, S., Zhang, X., and Wang, Y., 2011. “A time reversal focusing based impact imaging method and its evaluation on complex composite structures". Smart Materials and Structures, 20(10), p. 105014.

[12] Hall, J. S., and Michaels, J. E., 2015. "Multipath ultrasonic guided wave imaging in complex structures". Structural Health Monitoring, 14(4), pp. 345-358.

[13] Qiu, L., Liu, M., Qing, X., and Yuan, S., 2013. “A quantitative multidamage monitoring method for largescale complex composite". Structural Health Monitoring, 12(3), pp. 183-196.

[14] Michaels, J. E., 2008. "Detection, localization and characterization of damage in plates with an in situ array of spatially distributed ultrasonic sensors". Smart Materials and Structures, 17(035035).

[15] Hall, J. S., and E.Michaels, J., 2010. "Minimum variance ultrasonic imaging applied to an in situ sparse guided wave array". IEEE Transactions on Ultrasonics, Ferroelectrics, and Frequency Control, 57(10), pp. 2311-2323.

[16] Quaegebeur, N., Masson, P., Langlois-Demers, D., and Micheau, P., 2011. "Dispersion-based imaging for structural health monitoring using sparse and compact arrays". Smart Materials and Structures, 20(025005).

[17] Sharif-Khodaei, Z., and Aliabadi, M. H., 2014. "Assessment of delay-and-sum algorithms for damage detection in aluminium and composite plates". Smart Materials and Structures, 23(075007).

[18] Aldrin, J. C., Medina, E. A., Lingren, E. A., Buynak, C. F., and Knopp, J. S., 2011. Protocol for reliability assessment of structural health monitoring systems incorporating model-assisted probability of detection (mapod) approach. Tech. rep., DTIC Document.

[19] Kessler, S. S., Flynn, E. B., Dunn, C. T., and Todd, M. D., 2011. "A structural health monitoring software tool for optimization, diagnostics and prognostics". In Annual Conference of the Prognostics and Health Management Society.

[20] Etebu, E., and Shafiee, M., 2018. "Reliability analysis of strucutural health monitoring systems". In ESREL 
Proceedings: Safety and Reliability - Safe Societies in a Changing World.

[21] Schubert-Kabban, C. M., Greenwell, B. M., DeSimio, M. P., and Derriso, M. M., 2015. "The probability of detection for structural health monitoring systems: Repeated measures data”. Structural Health Monitoring, 14(3), pp. 252-264.

[22] Moriot, J., Quaegebeur, N., Le Duff, A., and Masson, P., 2016. "Characterization of the robustness of shm imaging techniques using the absolute error of localization". In Proceedings of the 8th European workshop on structural health monitoring, Bilbao.

[23] Kulakovskyi, A., Mesnil, O., Chapuis, B., d'Almeida, O., and Lhémery, A., 2019. "High-resolution defect imaging in laminate composites and honeycomb structures". In AIP Conference Proceedings, Vol. 2102, AIP Publishing LLC, p. 040008.

[24] Kausel, E., 1986. "Wave propagation in anisotropic layered media". International Journal for Numerical Methods in Engineering, 23(8), pp. 1567-1578.

[25] Hall, J. S., and Michaels, J. E., 2011. "Computational Efficiency of Ultrasonic Guided Wave Imaging Algorithms". IEEE Transactions on Ultrasonics, Ferroelectrics, and Frequency Control, 58(1), pp. 244-248.

[26] Quaegebeur, N., Micheau, P., Masson, P., and Maslouhi, A., 2010. "Structural health monitoring strategy for detection of interlaminar delamination in composite plates". Smart Materials and Structures, 19(085005).

[27] Zeng, L., Zhaoa, M., Lin, J., and Wu, W., 2016. "Waveform separation and image fusion for Lamb waves inspection resolution improvement". NDT \& E International, 79, pp. 17-29.

[28] Willberg, C., Duczek, S., Vivar-Perez, J. M., and Ahmad, Z. A. B., 2015. "Simulation methods for guided wave-based Structural Health Monitoring: A review". Applied Mechanics Reviews, 67(1), p. 010803.

[29] Leckey, C., Wheeler, K., Hafiychuk, V. N., Hafiychuk, H., and Timucin, D., 2018. "Simulation of guidedwave ultrasound propagation in composite laminates: Benchmark comparisons of numerical codes and experiment". Ultrasonics, 84, pp. 187-200.

[30] Imperiale, A., and Demaldent, E., 2019. "A macroelement strategy based upon spectral finite elements and mortar elements for transient wave propagation modeling. application to ultrasonic testing of laminate composite materials". International Journal for Numerical Methods in Engineering, 17(DOI: 10.1002/nme.6080).

[31] Cohen, G., 2002. Higher-Order Numerical Methods for Transient Wave Equations. Springer: Scientific Computation.

[32] Mesnil, O., Imperiale, A., Demaldent, E., and Chapuis, B., 2019. "Validation of spectral finite element simulation tools dedicated to structural health monitoring". AIP Conference Proceedings, 2102(050018).

[33] Chaabene, S., Bouchoucha, F., Ichchou, M. N., and M.Haddar, 2015. "Wave mode diffusion and propaga- tion in structural wave guide under varying temperature”. Applied Acoustics, 108, pp. 84-91.

[34] Miorelli, R., Kulakovskyi, A., Mesnil, O., Chapuis, B., and d'Almeida, O., 2020. "Supervised learning strategy for classification and regression tasks applied to aeronautical structural health monitoring problems". To be published (Currently Under Rewiew).

[35] Le Bourdais, F., Mesnil, O., and d'Almeida, O., 2019. "Machine-learning based temperature compensation for guided wave imaging in structural health monitoring”. In Proceedings of the 11th International Symposium on NDT in aerospace, Paris-Saclay,13-15th Nov. 2019.

[36] Fribourg-Blanc, E., 2003. "Thin film actuators for structural heath monitoring : study of pzt and pmnt films". PhD thesis, University of Valenciennes.

[37] Giurgiutiu, V., 2005. "Tuned lamb wave excitation and detection with piezoelectric wafer active sensors for structural health monitoring". Journal of intelligent material systems and structures, 16(4), pp. 291-305.

[38] Moriot, J., Quaegebeur, N., Duff, A. L., and Masson, P., 2017. "A model-based approach for statistical assessment of detection and localization performance of guided wave-based imaging techniques". Structural Health Monitoring, 17(6), pp. 1460-1472.

[39] Chen, Y.-C., 2017. "A tutorial on kernel density estimation and recent advances". Biostatistics \& Epidemiology, 1(1), pp. 161-187.

[40] Raschka, S., and Mirjalili, V., 2019. Python Machine Learning, 3rd Ed., 3 ed. Packt Publishing, Birmingham, UK. 